\title{
Pacific
}

Journal of

Mathematics

\section{ON YAMABE-TYPE PROBLEMS ON RIEMANNIAN MANIFOLDS WITH BOUNDARY}

Marco Ghimenti, Anna Maria Micheletti and Angela Pistoia 


\title{
ON YAMABE-TYPE PROBLEMS ON RIEMANNIAN MANIFOLDS WITH BOUNDARY
}

\author{
Marco Ghimenti, Anna Maria Micheletti and Angela Pistoia
}

Let $(M, g)$ be an $n$-dimensional compact Riemannian manifold with boundary. We consider the Yamabe-type problem

$$
\begin{cases}-\Delta_{g} u+a u=0 & \text { on } M, \\ \partial_{\nu} u+\frac{n-2}{2} b u=(n-2) u^{n /(n-2) \pm \varepsilon} & \text { on } \partial M,\end{cases}
$$

where $a \in C^{1}(M), b \in C^{1}(\partial M), v$ is the outward pointing unit normal to $\partial M$, $\Delta_{g} u:=\operatorname{div}_{g} \nabla_{g} u$, and $\varepsilon$ is a small positive parameter. We build solutions which blow up at a point of the boundary as $\varepsilon$ goes to zero. The blowing-up behavior is ruled by the function $b-H_{g}$, where $H_{g}$ is the boundary mean curvature.

\section{Introduction}

Let $(M, g)$ be a smooth, compact Riemannian manifold of dimension $n \geq 3$ with a boundary $\partial M$ which is the union of a finite number of smooth closed compact submanifolds embedded in $M$.

A well-known problem in differential geometry is whether $(M, g)$ is necessarily conformally equivalent to a manifold of constant scalar curvature whose boundary is minimal. When the boundary is empty this is called the Yamabe problem (see Yamabe [1960]), which has been completely solved by Aubin [1976], Schoen [1984] and Trudinger [1968]. Cherrier [1984] and Escobar [1992a; 1992b] studied the problem in the context of manifolds with boundary and gave an affirmative solution to the question in almost every case. The remaining cases were studied by Marques [2005; 2007], by Almaraz [2010] and by Brendle and Chen [2014].

Once the problem is solvable, a natural question about compactness of the full set of solutions arises. Concerning the Yamabe problem, it was first raised by Schoen in a topics course at Stanford University in 1988. A necessary condition is that the manifold is not conformally equivalent to the standard sphere $\mathbb{S}^{n}$, since the group of

The research that lead to the present paper was partially supported by the group GNAMPA of Istituto Nazionale di Alta Matematica (INdAM).

MSC2010: 35J20, 58J05.

Keywords: Yamabe problem, blowing-up solutions, compactness. 
conformal transformations of the round sphere is not compact itself. The problem of compactness has been widely studied in recent years and has been completely solved by Brendle [2008], Brendle and Marques [2009] and Khuri, Marques and Schoen [Khuri et al. 2009].

In the presence of a boundary, a necessary condition is that $M$ is not conformally equivalent to the standard ball $\mathbb{B}^{n}$. The problem when the boundary of the manifold is not empty has been studied by V. Felli and M. Ould Ahmedou [2003; 2005], Han and Li [1999] and Almaraz [2011a; 2011b]. In particular, Almaraz studied the compactness property in the case of scalar-flat metrics. Indeed, the zero scalar curvature case is particularly interesting because it leads one to study a linear equation in the interior with a critical Neumann-type nonlinear boundary condition

$$
\begin{cases}-\Delta_{g} u+\frac{n-2}{4(n-1)} R_{g} u=0 & \text { on } M, u>0 \text { in } M, \\ \partial_{\nu} u+\frac{n-2}{2} H_{g} u=(n-2) u^{n /(n-2)} & \text { on } \partial M,\end{cases}
$$

where $v$ is the outward pointing unit normal to $\partial M, R_{g}$ is the scalar curvature of $M$ with respect to $g$, and $H_{g}$ is the boundary mean curvature with respect to $g$.

We note that in this case compactness of solutions is equivalent to establish a priori estimates for solutions to equation (1-1). Almaraz [2011b] proved that compactness holds for a generic metric $g$. On the other hand, in [Almaraz 2011a] it was proved that if the dimension of the manifold is $n \geq 25$, compactness does not hold because it is possible to build blowing-up solutions to (1-1) for a suitable metric $g$. We point out that the problem of compactness in dimension $n \leq 24$ is still not completely understood.

An interesting issue, closely related to the compactness property, is the stability problem. One can ask whether or not the compactness property is preserved under perturbations of the equation, which is equivalent to having or not having uniform a priori estimates for solutions of the perturbed problem. Let us consider the more general problem

$$
\begin{cases}-\Delta_{g} u+a(x) u=0 & \text { in } M, u>0 \text { in } M, \\ \partial_{\nu} u+b(x) u=(n-2) u^{n /(n-2)} & \text { on } \partial M .\end{cases}
$$

We say that the problem (1-2) is stable if for any sequences of $C^{1}$ functions $a_{\varepsilon}: M \rightarrow \mathbb{R}$ and $b_{\varepsilon}: \partial M \rightarrow \mathbb{R}$ converging in $C^{1}$ to functions $a: M \rightarrow \mathbb{R}$ and $b: \partial M \rightarrow \mathbb{R}$, for any sequence of exponents $p_{\varepsilon}:=n /(n-2) \pm \varepsilon$ converging to the critical one $n /(n-2)$ and for any sequence of associated solutions $u_{\varepsilon}$ bounded in $H^{1}(M)$ of the perturbed problems

$$
\begin{cases}-\Delta_{g} u+a_{\varepsilon}(x) u=0 & \text { in } M, u_{\varepsilon}>0 \text { in } M, \\ \partial_{\nu} u+\frac{n-2}{2} b_{\varepsilon}(x) u=(n-2) u_{\varepsilon}^{n /(n-2) \pm \varepsilon} & \text { on } \partial M,\end{cases}
$$


there is a subsequence $u_{\varepsilon_{k}}$ which converges in $C^{2}$ to a solution to the limit problem (1-2). The stability of the Yamabe problem has been introduced and studied by Druet [2003; 2004] and by Druet and Hebey [2005a; 2005b]. Recently, Esposito, Pistoia and Vetois [Esposito et al. 2014], Micheletti, Pistoia and Vetois [Micheletti et al. 2009] and Esposito and Pistoia [2014] proved that a priori estimates fail for perturbations of the linear potential or of the exponent.

In this paper, we investigate the question of stability of the problem (1-2). It is clear that it is not stable if it is possible to build solutions $u_{\varepsilon}$ to perturbed problems (1-3) which blow up at one or more points of the manifold as the parameter $\varepsilon$ goes to zero. Here, we show that the behavior of the sequence $u_{\varepsilon}$ is dictated by the difference

$$
\varphi(q)=b(q)-H_{g}(q) \text { for } q \in \partial M .
$$

More precisely, we consider the problem

$$
\begin{cases}-\Delta_{g} u+a(x) u=0 & \text { on } M, u>0 \text { in } M, \\ \frac{\partial}{\partial \nu} u+\frac{n-2}{2} b(x) u=(n-2) u^{n /(n-2) \pm \varepsilon} & \text { on } \partial M .\end{cases}
$$

We assume that $a \in C^{1}(M)$ and $b \in C^{1}(\partial M)$ are such that the linear operator $\mathcal{L} u:=-\Delta_{g} u+a u$ with Neumann boundary condition $\mathcal{B} u:=\partial_{\nu} u+\frac{1}{2}(n-2) b u$ is coercive; namely, there exists a constant $c>0$ such that

$$
\int_{M}\left(\left|\nabla_{g} u\right|^{2}+a(x) u^{2}\right) d \mu_{g}+\frac{n-2}{2} \int_{\partial M} b(x) u^{2} d \sigma \geq c\|u\|_{H^{1}(M)}^{2} .
$$

Here $\varepsilon>0$ is a small parameter, $\Delta_{g} u:=\operatorname{div}_{g} \nabla_{g} u$, and the space $H^{1}(M)$ is the closure of $C^{\infty}(M)$ with respect to the norm

$$
\|u\|_{H^{1}}=\left(\int_{M}\left(\left|\nabla_{g} u\right|^{2}+u^{2}\right) d \mu_{g}\right)^{1 / 2} .
$$

The problem (1-5) turns out to be either slightly subcritical or slightly supercritical if the exponent in the nonlinearity is either $n /(n-2)-\varepsilon$ or $n /(n-2)+\varepsilon$, respectively. Let us state our main result.

Theorem 1. Assume (1-6) and $n \geq 7$.

(i) If $q_{0} \in \partial M$ is a strict local minimum point of the function $\varphi$ defined in (1-4) with $\varphi\left(q_{0}\right)>0$, then provided $\varepsilon>0$ is small enough, there exists a solution $u_{\varepsilon}$ of (1-5) in the slightly subcritical case such that $u_{\varepsilon}$ blows up at a boundary point when $\varepsilon \rightarrow 0^{+}$.

(ii) If $q_{0} \in \partial M$ is a strict local maximum point of the function $\varphi$ defined in (1-4) with $\varphi\left(q_{0}\right)<0$, then provided $\varepsilon<0$ is small enough, there exists a solution $u_{\varepsilon}$ of (1-5) in the slightly supercritical case such that $u_{\varepsilon}$ blows up at a boundary point when $\varepsilon \rightarrow 0^{+}$. 
We say that $u_{\varepsilon}$ blows up at a point $q_{0}$ of the boundary if there exists a family of points $q_{\varepsilon} \in \partial M$ such that $q_{\varepsilon} \rightarrow q_{0}$ as $\varepsilon \rightarrow 0$ and, for any neighborhood $U \subset M$ of $q_{0}$, we have that $\sup _{q \in U} u_{\varepsilon}(q) \rightarrow+\infty$ as $\varepsilon \rightarrow 0$.

Our result does not concern the stability of the geometric Yamabe problem (1-1). Indeed, the function $\varphi$ in (1-4) turns out to be identically zero. It would be interesting to discover the function which rules the behavior of blowing-up sequences in this case. We expect that it depends on the trace-free second fundamental form as it is suggested by Almaraz [2011b], where a compactness result in the subcritical case is established.

The case of low dimension also remains open, where we expect that the function $\varphi$ in (1-4) should be replaced by a function which depends on the Weyl tensor of the boundary, as suggested by Escobar [1992a; 1992b].

The proof of our result relies on a very well known Ljapunov-Schmidt procedure. In Section 2 we set up the problem, and in Section 3 we reduce the problem to a finite dimensional one, which is then studied in Section 4.

\section{Setting of the problem}

Let us rewrite problem (1-5) in a more convenient way.

First of all, assumption (1-6) allows us to endow the Hilbert space $H:=H^{1}(M)$ with the scalar product

$$
\langle\langle u, v\rangle\rangle_{H}:=\int_{M}\left(\nabla_{g} u \nabla_{g} v+a(x) u v\right) d \mu_{g}+\frac{n-2}{2} \int_{\partial M} b(x) u v d \sigma
$$

and the induced norm $\|u\|_{H}^{2}:=\langle\langle u, u\rangle\rangle_{H}$. We define the exponent

$$
s_{\varepsilon}= \begin{cases}\frac{2(n-1)}{n-2} & \text { in the subcritical case, } \\ \frac{2(n-1)}{n-2}+n \varepsilon & \text { in the supercritical case },\end{cases}
$$

and the Banach space $\mathcal{H}:=H^{1}(M) \cap L^{s_{\varepsilon}}(\partial M)$ endowed with the norm $\|u\|_{\mathcal{H}}=$ $\|u\|_{H}+|u|_{L^{s_{\varepsilon}}(\partial M)}$.

Notice that in the subcritical case $\mathcal{H}$ is identical to the Hilbert space $H$.

By trace theorems, we have the inclusion $W^{1, \tau}(M) \subset L^{t}(\partial M)$ for any $t$ and $\tau$ satisfying $t \leq \tau(n-1) /(n-\tau)$.

We consider $i: H^{1}(M) \rightarrow L^{2(n-1) /(n-2)}(\partial M)$ and its adjoint with respect to $\langle\langle\cdot, \cdot\rangle\rangle_{H}$, namely

$$
i^{*}: L^{2(n-1) / n}(\partial M) \rightarrow H^{1}(M)
$$

defined by

$$
\left\langle\left\langle\varphi, i^{*}(g)\right\rangle\right\rangle_{H}=\int_{\partial M} \varphi g d \sigma \quad \text { for all } \varphi \in H^{1}
$$


so that $u=i^{*}(g)$ is the weak solution of the problem

$$
\begin{cases}-\Delta_{g} u+a(x) u=0 & \text { on } M, \\ \frac{\partial}{\partial v} u+\frac{n-2}{2} b(x) u=g & \text { on } \partial M .\end{cases}
$$

We recall that by [Nittka 2011], if $u \in H^{1}(M)$ is a solution of (2-1), then for $2 n /(n+2) \leq q \leq n / 2$ and $r>0$ we have

$$
\|u\|_{L^{(n-1) q /(n-2 q)(\partial M)}}=\left\|i^{*}(g)\right\|_{L^{(n-1) q /(n-2 q)(\partial M)}} \leq\|g\|_{L^{(n-1) q /(n-q)+r}(\partial M)} .
$$

By this result, we can choose $q$ and $r$ such that

$$
\frac{(n-1) q}{n-2 q}=\frac{2(n-1)}{n-2}+n \varepsilon \quad \text { and } \quad \frac{(n-1) q}{n-q}+r=\frac{2(n-1)+n(n-2) \varepsilon}{n+(n-2) \varepsilon},
$$

that is,

$q=\frac{2 n+n^{2}\left(\frac{n-2}{n-1}\right) \varepsilon}{n+2+2 n\left(\frac{n-2}{n-1}\right) \varepsilon} \quad$ and $\quad r=\frac{2(n-1)+n(n-2) \varepsilon}{n+(n-2) \varepsilon}-\frac{2(n-1)+n(n-2) \varepsilon}{n+(n-2)\left(\frac{n}{n-1}\right) \varepsilon}$.

So, if $u \in L^{2(n-1) /(n-2)+n \varepsilon}(\partial M)$, then

$$
|u|^{\frac{n}{n-2}+\varepsilon} \in L^{\frac{2(n-1)+n(n-2) \varepsilon}{n+\varepsilon(n-2)}}(\partial M)
$$

and, in light of (2-2), also $i^{*}\left(|u|^{n /(n-2)+\varepsilon}\right) \in L^{2(n-1) /(n-2)+n \varepsilon}(\partial M)$.

Finally, we rewrite problem (1-5) - both in the subcritical and the supercritical case - as

$$
u=i^{*}\left(f_{\varepsilon}(u)\right), \quad u \in \mathcal{H},
$$

where the nonlinearity $f_{\varepsilon}(u)$ is defined as $f_{\varepsilon}(u):=(n-2)\left(u^{+}\right)^{n /(n-2)+\varepsilon}$ in the supercritical case or $f_{\varepsilon}(u):=(n-2)\left(u^{+}\right)^{n /(n-2)-\varepsilon}$ in the subcritical case. Here $u^{+}(x):=\max \{0, u(x)\}$. By assumption (1-6), a solution to problem (2-4) is strictly positive and actually is a solution to problem (1-5). Therefore, we are led to build solutions to problem (2-4) which blow-up at a boundary point as $\varepsilon$ goes to zero.

The main ingredient to cook up our solutions are the standard bubbles

$$
U_{\delta, \xi}(x, t):=\frac{\delta^{(n-2) / 2}}{\left((\delta+t)^{2}+|x-\xi|^{2}\right)^{(n-2) / 2}}, \quad(x, t) \in \mathbb{R}^{n-1} \times \mathbb{R}_{+}, \delta>0, \xi \in \mathbb{R}^{n-1},
$$

which are all the solutions to the limit problem

$$
\begin{cases}-\Delta U=0 & \text { on } \mathbb{R}^{n-1} \times \mathbb{R}_{+} \\ \partial_{\nu} U=(n-2) U^{n /(n-2)} & \text { on } \mathbb{R}^{n-1} \times\{t=0\} .\end{cases}
$$

We set $U_{\delta}(x, t):=U_{\delta, 0}(x, t)$. 
We also need to introduce the linear problem

$$
\begin{cases}-\Delta V=0 & \text { on } \mathbb{R}^{n-1} \times \mathbb{R}_{+}, \\ \partial_{v} V=n U_{1}^{2 /(n-2)} V & \text { on } \mathbb{R}^{n-1} \times\{t=0\} .\end{cases}
$$

In [Almaraz 2011b] it has been proved that the $n$-dimensional space of solutions of (2-6) is generated by the functions

$$
\begin{aligned}
& V_{i}=\frac{\partial U_{1}}{\partial x_{i}}=(2-n) \frac{x_{i}}{\left((1+t)^{2}+|x|^{2}\right)^{n / 2}} \quad \text { for } i=i, \ldots, n-1, \\
& V_{0}=\left.\frac{\partial U_{\delta}}{\partial \delta}\right|_{\delta=1}=\frac{n-2}{2}\left(\frac{1}{(1+t)^{2}+|x|^{2}}\right)^{n / 2}\left(t^{2}+|x|^{2}-1\right) .
\end{aligned}
$$

Next, for a point $q \in \partial M$ and the $(n-1)$-dimensional unitary ball $B^{n-1}(0, R)$ in $\mathbb{R}^{n-1}$, we introduce the Fermi coordinates $\psi_{q}^{\partial}: B^{n-1}(0, R) \times[0, R) \rightarrow M$. We read the bubble on the manifold as the function

$$
W_{\delta, q}(\xi)=U_{\delta}\left(\left(\psi_{q}^{\partial}\right)^{-1} \xi\right) \chi\left(\left(\psi_{q}^{\partial}\right)^{-1} \xi\right),
$$

and the functions $V_{i}$ on the manifold as the functions

$$
Z_{\delta, q}^{i}(\xi)=\frac{1}{\delta^{(n-2) / 2}} V_{i}\left(\frac{1}{\delta}\left(\psi_{q}^{\partial}\right)^{-1} \xi\right) \chi\left(\left(\psi_{q}^{\partial}\right)^{-1} \xi\right) \quad \text { for } i=0, \ldots, n-1,
$$

where $\chi(x, t)=\tilde{\chi}(|x|) \tilde{\chi}(t)$, for $\tilde{\chi}$ a smooth cut off function, $\tilde{\chi}(s) \equiv 1$ for $0 \leq s<$ $R / 2$ and $\tilde{\chi}(s) \equiv 0$ for $s \geq R$. Then, it is necessary to split the Hilbert space $H$ into the sum of the orthogonal spaces

$$
K_{\delta, q}=\operatorname{Span}\left\langle Z_{\delta, q}^{0}, \ldots, Z_{\delta, q}^{n-1}\right\rangle
$$

and

$$
K_{\delta, q}^{\perp}=\left\{\varphi \in H^{1}(M) \mid\left\langle\left\langle\varphi, Z_{\delta, q}^{i}\right\rangle\right\rangle_{H}=0 \text { for all } i=0, \ldots, n-1\right\} .
$$

Finally, we can look for a solution to problem (2-4) in the form

$$
u_{\varepsilon}(x)=W_{\delta, q}(x)+\phi(x)
$$

where the blow-up point $q$ is in $\partial M$, the blowing-up rate $\delta$ satisfies

$$
\delta:=d \varepsilon \quad \text { for some } d>0
$$

and the remainder term $\phi$ belongs to the infinite dimensional space $K_{\delta, q}^{\perp} \cap \mathcal{H}$ of codimension $n$. We are led to solve the system

$$
\begin{aligned}
& \Pi_{\delta, q}^{\perp}\left\{W_{\delta, q}(x)+\phi(x)-i^{*}\left(f_{\varepsilon}\left(W_{\delta, q}(x)+\phi(x)\right)\right)\right\}=0, \\
& \Pi_{\delta, q}\left\{W_{\delta, q}(x)+\phi(x)-i^{*}\left(f_{\varepsilon}\left(W_{\delta, q}(x)+\phi(x)\right)\right)\right\}=0,
\end{aligned}
$$

$\Pi_{\delta, q}^{\perp}$ and $\Pi_{\delta, q}$ being the projections on $K_{\delta, q}^{\perp}$ and $K_{\delta, q}$, respectively. 


\section{The finite dimensional reduction}

In this section we perform the finite dimensional reduction. We rewrite the auxiliary equation (2-8) in the equivalent form

$$
L(\phi)=N(\phi)+R,
$$

where $L=L_{\delta, q}: K_{\delta, q}^{\perp} \cap \mathcal{H} \rightarrow K_{\delta, q}^{\perp} \cap \mathcal{H}$ is the linear operator

$$
L(\phi)=\Pi_{\delta, q}^{\perp}\left\{\phi(x)-i^{*}\left(f_{\varepsilon}^{\prime}\left(W_{\delta, q}\right)[\phi]\right)\right\},
$$

$N(\phi)$ is the nonlinear term

$$
N(\phi)=\Pi_{\delta, q}^{\perp}\left\{i^{*}\left(f_{\varepsilon}\left(W_{\delta, q}(x)+\phi(x)\right)\right)-i^{*}\left(f_{\varepsilon}\left(W_{\delta, q}(x)\right)\right)-i^{*}\left(f_{\varepsilon}^{\prime}\left(W_{\delta, q}\right)[\phi]\right)\right\}
$$

and the error term $R$ is defined by

$$
R=\Pi_{\delta, q}^{\perp}\left\{i^{*}\left(f_{\varepsilon}\left(W_{\delta, q}(x)\right)\right)-W_{\delta, q}(x)\right\} .
$$

\subsection{The invertibility of the linear operator $L$.}

Lemma 2. For $a, b \in \mathbb{R}$ with $0<a<b$, there exists a positive constant $C_{0}=C_{0}(a, b)$ such that, for $\varepsilon$ small, for any $q \in \partial M$, for any $d \in[a, b]$ and for any $\phi \in K_{\delta, q}^{\perp} \cap \mathcal{H}$, we have

$$
\left\|L_{\delta, q}(\phi)\right\|_{\mathcal{H}} \geq C_{0}\|\phi\|_{\mathcal{H}} .
$$

Proof. We argue by contradiction. Suppose that there exist two sequences of real numbers $\varepsilon_{m} \rightarrow 0$ and $d_{m} \in[a, b]$, a sequence of points $q_{m} \in \partial M$ and a sequence of functions $\phi_{\varepsilon_{m}} d_{m}, q_{m} \in K_{\varepsilon_{m}}^{\perp} d_{m}, q_{m} \cap \mathcal{H}$ such that

$$
\left\|\phi_{\varepsilon_{m} d_{m}, q_{m}}\right\|_{\mathcal{H}}=1 \quad \text { and } \quad\left\|L_{\varepsilon_{m} d_{m}, q_{m}}\left(\phi_{\varepsilon_{m} d_{m}, q_{m}}\right)\right\|_{\mathcal{H}} \rightarrow 0 \text { as } m \rightarrow+\infty \text {. }
$$

For the sake of simplicity, we set $\delta_{m}=\varepsilon_{m} d_{m}$ and define

$$
\tilde{\phi}_{m}:=\delta_{m}^{(n-2) / 2} \phi_{\delta_{m}, q_{m}}\left(\psi_{q_{m}}^{\partial}\left(\delta_{m} \eta\right)\right) \chi\left(\delta_{m} \eta\right) \quad \text { for } \eta=(z, t) \in \mathbb{R}_{+}^{n}, z \in \mathbb{R}^{n-1}, t \geq 0 .
$$

Since $\left\|\phi_{\varepsilon_{m}} d_{m}, q_{m}\right\|_{H} \leq 1$, by a change of variables we easily get that $\left\{\tilde{\phi}_{m}\right\}_{m}$ is bounded in $D^{1,2}\left(\mathbb{R}_{+}^{n}\right)$ (but not in $H^{1}\left(\mathbb{R}_{+}^{n}\right)$ ). Therefore, there exists $\tilde{\phi} \in D^{1,2}\left(\mathbb{R}_{+}^{n}\right)$ such that $\tilde{\phi}_{m} \rightarrow \tilde{\phi}$ almost everywhere, weakly in $D^{1,2}\left(\mathbb{R}_{+}^{n}\right)$, in $L^{2 n /(n-2)}\left(\mathbb{R}_{+}^{n}\right)$ and strongly in $L_{\mathrm{loc}}^{2(n-1) /(n-2)}\left(\partial \mathbb{R}_{+}^{n}\right)$.

Since $\phi_{\delta_{m}}, q_{m} \in K_{\delta_{m}}^{\perp}, q_{m}$, and taking (2-6) into account, for $i=0, \ldots, n-1$ we get

$$
o(1)=\int_{\mathbb{R}_{+}^{n}} \nabla \tilde{\phi} \nabla V_{i} d z d t=n \int_{\mathbb{R}^{n-1}} U_{1}^{2 /(n-2)}(z, 0) V_{i}(z, 0) \tilde{\phi}(z, 0) d z
$$


Indeed, by a change of variables we have

$$
\begin{aligned}
& 0=\left\langle\left\langle\phi_{\delta_{m}, q_{m}}, Z_{\delta_{m}, q_{m}}^{i}\right\rangle_{H}\right. \\
& =\int_{M}\left(\nabla_{g} \phi_{\delta_{m}, q_{m}} \nabla_{g} Z_{\delta_{m}, q_{m}}^{i}+a(x) \phi_{\delta_{m}, q_{m}} Z_{\delta_{m}, q_{m}}^{i}\right) d \mu_{g} \\
& +\frac{n-2}{2} \int_{\partial M} b(x) \phi_{\delta_{m}, q_{m}} Z_{\delta_{m}, q_{m}}^{i} d \sigma \\
& =\int_{\mathbb{R}_{+}^{n}}\left|g_{q_{m}}(\delta \eta)\right|^{1 / 2} \delta^{(n-2) / 2} g_{q_{m}}^{\alpha \beta}(\delta \eta) \frac{\partial}{\partial \eta_{\alpha}} V_{i}(\eta) \chi(\delta \eta) \frac{\partial}{\partial \eta_{\alpha}} \phi_{\delta_{m}, q_{m}}\left(\psi_{q_{m}}^{\partial}\left(\delta_{m} \eta\right)\right) \delta \eta \\
& +\int_{\mathbb{R}_{+}^{n}}\left|g_{q_{m}}(\delta \eta)\right|^{1 / 2} \delta^{(n+2) / 2} a\left(\psi_{q_{m}}^{\partial}(\delta \eta)\right) V_{i}(\eta) \phi_{\delta_{m}, q_{m}}\left(\psi_{q_{m}}^{\partial}\left(\delta_{m} \eta\right)\right) \delta \eta \\
& +\int_{\partial \mathbb{R}_{+}^{n}}\left|g_{q_{m}}(\delta z, 0)\right|^{1 / 2} \delta^{n / 2} b\left(\psi_{q_{m}}^{\partial}(\delta \eta)\right) \phi_{\delta_{m}, q_{m}}\left(\psi_{q_{m}}^{\partial}\left(\delta_{m} z, 0\right)\right) V_{i}\left(\delta_{m} z, 0\right) d z \\
& =\int_{\mathbb{R}_{+}^{n}} \nabla V_{i}(\eta) \nabla \tilde{\phi}_{m}(\eta)+\delta^{2} a\left(q_{m}\right) V_{i}(\eta) \tilde{\phi}_{m}(\eta) \delta \eta \\
& +\delta \int_{\partial \mathbb{R}_{+}^{n}} b\left(q_{m}\right) V_{i}(z, 0) \tilde{\phi}_{m}(z, 0) \delta \eta+O(\delta) \\
& =\int_{\mathbb{R}_{+}^{n}} \nabla V_{i}(\eta) \nabla \tilde{\phi}_{m}(\eta)+O(\delta)=\int_{\mathbb{R}_{+}^{n}} \nabla V_{i}(\eta) \nabla \tilde{\phi}(\eta)+o(1),
\end{aligned}
$$

By definition of $L_{\delta_{m}, q_{m}}$ we have

$$
\phi_{\delta_{m}, q_{m}}-i^{*}\left(f_{\varepsilon}^{\prime}\left(W_{\delta_{m}, q_{m}}\right)\left[\phi_{\delta_{m}, q_{m}}\right]\right)-L_{\delta_{m}, q_{m}}\left(\phi_{\delta_{m}, q_{m}}\right)=\sum_{i=0}^{n-1} c_{m}^{i} Z_{\delta_{m}, q_{m}}^{i} .
$$

We want to prove that, for all $i=0, \ldots, n-1, c_{m}^{i} \rightarrow 0$ as $m \rightarrow \infty$. Multiplying (3-5) by $Z_{\delta_{m}, q_{m}}^{j}$ we obtain, by definition of $i^{*}$,

$$
\begin{aligned}
\sum_{i=0}^{n-1} c_{m}^{i}\left\langle\left\langle Z_{\delta_{m}, q_{m}}^{i}, Z_{\delta_{m}, q_{m}}^{j}\right\rangle_{H}\right. & =\left\langle\left\langle i^{*}\left(f_{\varepsilon_{m}}^{\prime}\left(W_{\delta_{m}, q_{m}}\right)\left[\phi_{\delta_{m}, q_{m}}\right]\right), Z_{\delta_{m}, q_{m}}^{j}\right\rangle_{H}\right. \\
& =\int_{\partial M} f_{\varepsilon_{m}}^{\prime}\left(W_{\delta_{m}, q_{m}}\right)\left[\phi_{\delta_{m}, q_{m}}\right] Z_{\delta_{m}, q_{m}}^{j} d \sigma .
\end{aligned}
$$

Moreover, by multiplying (3-5) by $\phi_{\delta_{m}, q_{m}}$ we obtain that

$$
\left\|\phi_{\delta_{m}, q_{m}}\right\|_{H}-\int_{\partial M} f_{\varepsilon_{m}}^{\prime}\left(W_{\delta_{m}, q_{m}}\right) \phi_{\delta_{m}, q_{m}}^{2} d \sigma \rightarrow 0
$$

Thus $\left(f_{\varepsilon_{m}}^{\prime}\left(W_{\delta_{m}, q_{m}}\right)\right)^{1 / 2} \phi_{\delta_{m}, q_{m}}$ is bounded and weakly convergent in $L^{2}(\partial M)$. With this consideration we easily get 


$$
\begin{aligned}
\int_{\partial M} f_{\varepsilon_{m}}^{\prime}\left(W_{\delta_{m}, q_{m}}\right)\left[\phi_{\delta_{m}, q_{m}}\right] & Z_{\delta_{m}, q_{m}}^{j} d \sigma \\
= & \int_{\partial M}\left(f_{\varepsilon_{m}}^{\prime}\left(W_{\delta_{m}, q_{m}}\right)\right)^{1 / 2} \phi_{\delta_{m}, q_{m}}\left(f_{\varepsilon_{m}}^{\prime}\left(W_{\delta_{m}, q_{m}}\right)\right)^{1 / 2} Z_{\delta_{m}, q_{m}}^{j} d \sigma \\
& =n \int_{\mathbb{R}^{n-1}} U_{1}^{2 /(n-2)}(z, 0) \tilde{\phi}(z, 0) V_{i}(z, 0) d z+o(1)=o(1),
\end{aligned}
$$

once we take (3-4) into account.

Now, it is easy to prove that

$$
\left\langle\left\langle Z_{\delta_{m}, q_{m}}^{i}, Z_{\delta_{m}, q_{m}}^{j}\right\rangle_{H}=C \delta_{i j}+o(1),\right.
$$

hence we can conclude that $c_{m}^{i} \rightarrow 0$ as $m \rightarrow \infty$ for each $i=0, \ldots, n-1$. This, combined with (3-5) and using $\left\|L_{\varepsilon_{m} d_{m}, q_{m}}\left(\phi_{\varepsilon_{m} d_{m}, q_{m}}\right)\right\|_{\mathcal{H}} \rightarrow 0$, gives us that

$$
\left\|\phi_{\delta_{m}, q_{m}}-i^{*}\left(f_{\mathcal{E}}^{\prime}\left(W_{\delta_{m}, q_{m}}\right)\left[\phi_{\delta_{m}, q_{m}}\right]\right)\right\|_{\mathcal{H}}=\sum_{i=0}^{n-1} c_{m}^{i}\left\|Z^{i}\right\|_{\mathcal{H}}+o(1)=o(1) .
$$

Choose a smooth function $\varphi \in C_{0}^{\infty}\left(\mathbb{R}_{+}^{n}\right)$ and define

$$
\varphi_{m}(x)=\frac{1}{\delta_{m}^{(n-2) / 2}} \varphi\left(\frac{1}{\delta_{m}}\left(\psi_{q_{m}}^{\partial}\right)^{-1}(x)\right) \chi\left(\left(\psi_{q_{m}}^{\partial}\right)^{-1}(x)\right) \text { for } x \in M .
$$

We have that $\left\|\varphi_{m}\right\|_{H}$ is bounded and, by (3-6), that

$$
\begin{aligned}
& \left\langle\left\langle\phi_{\delta_{m}, q_{m}}, \varphi_{m}\right\rangle\right\rangle_{H} \\
& =\int_{\partial M} f_{\varepsilon_{m}}^{\prime}\left(W_{\delta_{m}, q_{m}}\right)\left[\phi_{\delta_{m}, q_{m}}\right] \varphi_{m} d \sigma+\left\langle\left\langle\phi_{\delta_{m}, q_{m}}-i^{*}\left(f_{\varepsilon_{m}}^{\prime}\left(W_{\delta_{m}, q_{m}}\right)\left[\phi_{\delta_{m}, q_{m}}\right]\right), \varphi_{m}\right\rangle\right\rangle_{H} \\
& =\int_{\partial M} f_{\varepsilon_{m}}^{\prime}\left(W_{\delta_{m}, q_{m}}\right)\left[\phi_{\delta_{m}, q_{m}}\right] \varphi_{m} d \sigma+o(1) \\
& =\left(n \pm \varepsilon_{m}(n-2)\right) \int_{\mathbb{R}^{n-1}} \frac{1}{\delta_{m}^{ \pm \varepsilon n /(n-2)}} U_{1}^{2 /(n-2) \pm \varepsilon_{m}}(z, 0) \tilde{\phi}_{m}(z, 0) \varphi d z+o(1) \\
& =n \int_{\mathbb{R}^{n-1}} U_{1}^{2 /(n-2)}(z, 0) \tilde{\phi}(z, 0) \varphi(z, 0) d z+o(1),
\end{aligned}
$$

by the strong $L_{\text {loc }}^{2(n-1) /(n-2)}\left(\partial \mathbb{R}_{+}^{n}\right)$ convergence of $\tilde{\phi}_{m}$. On the other hand,

$$
\left\langle\left\langle\phi_{\delta_{m}, q_{m}}, \varphi_{m}\right\rangle\right\rangle_{H}=\int_{\mathbb{R}_{+}^{n}} \nabla \tilde{\phi} \nabla \varphi \delta \eta+o(1)
$$

so $\tilde{\phi}$ is a weak solution of (2-5) and we conclude that

$$
\tilde{\phi} \in \operatorname{Span}\left\{V_{0}, V_{1}, \ldots, V_{n}\right\} .
$$


This, combined with (3-4), gives that $\tilde{\phi}=0$. Proceeding as before we have

$$
\begin{aligned}
& \left\langle\left\langle\phi_{\delta_{m}, q_{m}}, \phi_{\delta_{m}, q_{m}}\right\rangle\right\rangle_{H} \\
& =\int_{\partial M} f_{\varepsilon_{m}}^{\prime}\left(W_{\delta_{m}, q_{m}}\right)\left[\phi_{\delta_{m}, q_{m}}\right] \phi_{\delta_{m}, q_{m}} d \sigma+o(1) \\
& =\left(n \pm \varepsilon_{m}(n-2)\right) \int_{\mathbb{R}^{n-1}} \frac{1}{\delta_{m}^{ \pm \varepsilon n /(n-2)}} U_{1}^{2 /(n-2) \pm \varepsilon_{m}}(z, 0) \tilde{\phi}_{m}^{2}(z, 0) \varphi d z+o(1)=o(1) .
\end{aligned}
$$

In a similar way, by (3-6) we have

$$
\left|\phi_{\delta_{m}}, q_{m}\right|_{L^{s_{\varepsilon}}}=\left|i^{*}\left(f_{\varepsilon}^{\prime}\left(W_{\delta_{m}}, q_{m}\right)\left[\phi_{\delta_{m}, q_{m}}\right]\right)\right|_{L^{s_{\varepsilon}}}+o(1)=o(1),
$$

which gives $\left\|\phi_{\delta_{m}, q_{m}}\right\|_{\mathcal{H}} \rightarrow 0$, which is a contradiction.

\subsection{The estimate of the error term $R$.}

Lemma 3. For $a, b \in \mathbb{R}$ with $0<a<b$, there exists a positive constant $C_{1}=C_{1}(a, b)$ such that, for $\varepsilon$ small, for any $q \in \partial M$ and for any $d \in[a, b]$ we have

$$
\left\|R_{\mathcal{E}, \delta, q}\right\|_{\mathcal{H}} \leq C_{1} \varepsilon|\ln \varepsilon|
$$

Proof. We estimate

$$
\begin{aligned}
& \left\|i^{*}\left(f_{\varepsilon}\left(W_{\delta, q}(x)\right)\right)-W_{\delta, q}(x)\right\|_{H} \\
& \quad \leq\left\|i^{*}\left(f_{\varepsilon}\left(W_{\delta, q}(x)\right)\right)-i^{*}\left(f_{0}\left(W_{\delta, q}(x)\right)\right)\right\|_{H}+\left\|i^{*}\left(f_{0}\left(W_{\delta, q}(x)\right)\right)-W_{\delta, q}(x)\right\|_{H} .
\end{aligned}
$$

By definition of $i^{*}$ there exists $\Gamma$ which solves the equation

$$
\begin{cases}-\Delta_{g} \Gamma+a(x) \Gamma=0 & \text { on } M, \\ \frac{\partial}{\partial v} \Gamma+\frac{n-2}{2} b(x) \Gamma=f_{0}\left(W_{\delta, q}\right) & \text { on } \partial M,\end{cases}
$$

so, by (3-7), we have

$$
\begin{aligned}
& \left\|i^{*}\left(f_{0}\left(W_{\delta, q}(x)\right)\right)-W_{\delta, q}(x)\right\|_{H} \\
& =\left\|\Gamma(x)-W_{\delta, q}(x)\right\|_{H}^{2} \\
& =\int_{M}\left[-\Delta_{g}\left(\Gamma-W_{\delta, q}\right)+a\left(\Gamma-W_{\delta, q}\right)\right]\left(\Gamma-W_{\delta, q}\right) d \mu_{g} \\
& \quad \quad+\int_{\partial M}\left[\frac{\partial}{\partial v}\left(\Gamma-W_{\delta, q}\right)+\frac{(n-2)}{2} b(x)\left(\Gamma-W_{\delta, q}\right)\right]\left(\Gamma-W_{\delta, q}\right) d \mu_{g} \\
& =\int_{M}\left[\Delta_{g} W_{\delta, q}-a W_{\delta, q}\right]\left(\Gamma-W_{\delta, q}\right) d \mu_{g} \\
& \quad+\int_{\partial M}\left[f_{0}\left(W_{\delta, q}\right)-\frac{\partial}{\partial v} W_{\delta, q}\right]\left(\Gamma-W_{\delta, q}\right) d \mu_{g} \\
& \quad-\frac{n-2}{2} \int_{\partial M} b(x) W_{\delta, q}\left(\Gamma-W_{\delta, q}\right) d \mu_{g}:=I_{1}+I_{2}+I_{3} .
\end{aligned}
$$


We obtain

$$
I_{1}=\left\|\Gamma-W_{\delta, q}\right\|_{H} O(\delta) .
$$

In fact,

$$
\begin{aligned}
I_{1} & \leq\left|\Delta_{g} W_{\delta, q}-a W_{\delta, q}\right|_{L^{2 n /(n+2)}(M)}\left|\Gamma-W_{\delta, q}\right|_{L^{2 n /(n-2)}(M)} \\
& \leq\left|\Delta_{g} W_{\delta, q}-a W_{\delta, q}\right|_{L^{2 n /(n+2)}(M)}\left\|\Gamma-W_{\delta, q}\right\|_{H} .
\end{aligned}
$$

We easily have that $\left|W_{\delta, q}\right|_{L^{2 n /(n+2)}}=O\left(\delta^{2}\right)$. For the other term we have, in coordinates,

$$
\Delta_{g} W_{\delta, q}=\Delta\left[U_{\delta} \chi\right]+\left(g^{a b}-\delta_{a b}\right) \partial_{a b}\left[U_{\delta} \chi\right]-g^{a b} \Gamma_{a b}^{k} \partial_{k}\left[U_{\delta} \chi\right],
$$

$\Gamma_{a b}^{k}$ being the Christoffel symbols. Using the expansion of the metric $g^{a b}$ given by (4-2) and (4-3) we have that

$$
\begin{aligned}
\left|\left(g^{a b}-\delta_{a b}\right) \partial_{a b}\left[U_{\delta} \chi\right]\right|_{L^{2 n /(n+2)}(M)} & =O(\delta), \\
\left|g^{a b} \Gamma_{a b}^{k} \partial_{k}\left[U_{\delta} \chi\right]\right|_{L^{2 n /(n+2)}(M)} & =O\left(\delta^{2}\right) .
\end{aligned}
$$

Since $U_{\delta}$ is a harmonic function we deduce

$$
\left|\Delta\left[U_{\delta} \chi\right]\right|_{L^{2 n /(n+2)}(M)}=\left|U_{\delta} \Delta \chi+2 \nabla U_{\delta} \nabla \chi\right|_{L^{2 n /(n+2)}(M)}=O\left(\delta^{2}\right) .
$$

For the second integral $I_{2}$ we have

$$
I_{2}=\left\|\Gamma-W_{\delta, q}\right\|_{H} O\left(\delta^{2}\right),
$$

since

$$
\begin{aligned}
I_{2} & \leq\left|f_{0}\left(W_{\delta, q}\right)-\frac{\partial}{\partial v} W_{\delta, q}\right|_{L^{2(n-1) / n}(\partial M)}\left|\Gamma-W_{\delta, q}\right|_{L^{2(n-1) / n-2}(\partial M)} \\
& \leq C\left|f_{0}\left(W_{\delta, q}\right)-\frac{\partial}{\partial v} W_{\delta, q}\right|_{L^{2(n-1) / n}(\partial M)}\left\|\Gamma-W_{\delta, q}\right\|_{H},
\end{aligned}
$$

and, using the boundary condition for (2-5), we have

$$
\begin{gathered}
\left|f_{0}\left(W_{\delta, q}\right)-\frac{\partial}{\partial \nu} W_{\delta, q}\right|_{L^{2(n-1) / n}(\partial M)} \\
=\frac{1}{\delta^{n / 2}}\left(\int _ { \mathbb { R } ^ { n - 1 } } | g ( \delta z , 0 ) | ^ { 1 / 2 } \left[(n-2) U^{n /(n-2)}(z, 0) \chi^{n /(n-2)}(\delta z, 0)\right.\right. \\
\left.\left.-\chi(\delta z, 0) \frac{\partial U}{\partial t}(z, 0)\right]^{\frac{2(n-1)}{n}} \delta^{n-1} d z\right)^{\frac{n}{2(n-1)}} \\
\leq C\left(\int _ { \mathbb { R } ^ { n - 1 } } \left[( n - 2 ) U ^ { n / ( n - 2 ) } ( z , 0 ) \left[\chi^{n /(n-2)}(\delta z, 0)\right.\right.\right. \\
\left.-\chi(\delta z, 0)]]^{\frac{2(n-1)}{n}} d z\right)^{\frac{n}{2(n-1)}}=O\left(\delta^{2}\right) .
\end{gathered}
$$


Lastly,

$$
I_{3} \leq\left|W_{\delta, q}\right|_{L^{2(n-1) / n}(\partial M)}\left|\Gamma-W_{\delta, q}\right|_{L^{2(n-1) /(n-2)}(\partial M)}=\left\|\Gamma-W_{\delta, q}\right\|_{H} O(\delta) .
$$

By (3-8), (3-12) and (3-14) we conclude that

$$
\left\|i^{*}\left(f_{0}\left(W_{\delta, q}(x)\right)\right)-W_{\delta, q}(x)\right\|_{H}=\left\|\Gamma(x)-W_{\delta, q}(x)\right\|_{H}=O(\delta) .
$$

To conclude the proof we estimate the term $\left\|i^{*}\left(f_{\varepsilon}\left(W_{\delta, q}(x)\right)\right)-i^{*}\left(f_{0}\left(W_{\delta, q}(x)\right)\right)\right\|_{H}$. We have, by the properties of $i^{*}$, that

$$
\begin{aligned}
& \left\|i^{*}\left(f_{\varepsilon}\left(W_{\delta, q}(x)\right)\right)-i^{*}\left(f_{0}\left(W_{\delta, q}(x)\right)\right)\right\|_{H} \\
& \leq\left|W_{\delta, q}(x)^{n /(n-2) \pm \varepsilon}-W_{\delta, q}^{n /(n-2)}(x)\right|_{L^{2(n-1) / n}(\partial M)} \\
& =\left(\int_{\mathbb{R}^{n-1}}\left[\left(\frac{1}{\delta^{ \pm \varepsilon(n-2) / 2}} U^{ \pm \varepsilon}(z, 0)-1\right) U^{n /(n-2)}(z, 0)\right]^{\frac{2(n-1)}{n}} d z\right)^{\frac{n}{2(n-1)}}+O\left(\delta^{2}\right) .
\end{aligned}
$$

To estimate the last integral, we first recall two Taylor expansions with respect to $\varepsilon$ :

$$
\begin{aligned}
U^{ \pm \varepsilon} & =1 \pm \varepsilon \ln U+\frac{1}{2} \varepsilon^{2} \ln ^{2} U+o\left(\varepsilon^{2}\right), \\
\delta^{\mp \varepsilon(n-2) / 2} & =1 \mp \varepsilon \frac{n-2}{2} \ln \delta+\varepsilon^{2} \frac{(n-2)^{2}}{8} \ln ^{2} \delta+o\left(\varepsilon^{2} \ln ^{2} \delta\right) .
\end{aligned}
$$

In light of (3-15) and (3-16) we have

$$
\begin{aligned}
& \left\|i^{*}\left(f_{\varepsilon}\left(W_{\delta, q}\right)\right)-i^{*}\left(f_{0}\left(W_{\delta, q}\right)\right)\right\|_{H} \\
& \leq\left(\int_{\mathbb{R}^{n-1}} \mid\left(\mp \frac{n-2}{2} \varepsilon \ln \delta \pm \varepsilon \ln U(z, 0)+O\left(\varepsilon^{2}\right)\right.\right. \\
& \left.\left.\quad+O\left(\varepsilon^{2} \ln \delta\right)\right)\left.U^{n /(n-2)}(z, 0)\right|^{\frac{2(n-1)}{n}} d z\right)^{\frac{n}{2(n-1)}}+O\left(\delta^{2}\right) \\
& =\frac{n-2}{2} \varepsilon \ln \delta|U(z, 0)|_{L^{2(n-1) /(n-2)}\left(\mathbb{R}^{n-1}\right)}^{n /(n-2)} \\
& \quad+\varepsilon\left(\int_{\mathbb{R}^{n-1}} U^{2(n-1) /(n-2)}(z, 0) \ln U(z, 0) d z\right)^{\frac{n}{2(n-1)}} \\
& =O(\varepsilon)+O(\varepsilon|\ln \delta|)+O\left(\delta^{2}\right) .
\end{aligned}
$$

Choosing $\delta=d \varepsilon$ concludes the proof of Lemma 3 for the subcritical case. 
For the supercritical case, we have to control $\left|R_{\varepsilon, \delta, q}\right|_{L^{s_{\varepsilon}}(\partial M)}$. As in the previous case we consider

$$
\begin{aligned}
\left|R_{\varepsilon, \delta, q}\right|_{L^{s_{\varepsilon}}(\partial M)} \leq \mid i^{*}\left(f_{\varepsilon}\left(W_{\delta, q}(x)\right)\right)-i^{*}\left(f_{0}(\right. & \left.\left.W_{\delta, q}(x)\right)\right)\left.\right|_{L^{s_{\varepsilon}}(\partial M)} \\
& +\left|i^{*}\left(f_{0}\left(W_{\delta, q}(x)\right)\right)-W_{\delta, q}(x)\right|_{L^{s_{\varepsilon}(\partial M)}} .
\end{aligned}
$$

As before, set $\Gamma=i^{*}\left(f_{0}\left(W_{\delta, q}(x)\right)\right.$. Since $\Gamma$ solves (3-7), $\Gamma-W_{\delta, q}$ solves

$$
\left\{\begin{array}{rr}
-\Delta_{g}\left(\Gamma-W_{\delta, q}\right)+a(x)\left(\Gamma-W_{\delta, q}\right)=-\Delta_{g} W_{\delta, q}+a(x) W_{\delta, q} & \text { on } M, \\
\frac{\partial}{\partial v}\left(\Gamma-W_{\delta, q}\right)+\frac{n-2}{2} b(x)\left(\Gamma-W_{\delta, q}\right) & \\
=f_{0}(\Gamma)+\frac{\partial}{\partial v} W_{\delta, q}+\frac{n-2}{2} b(x) W_{\delta, q} &
\end{array}\right.
$$

We choose $q$ as in (2-3), and $r=\varepsilon$. Thus, by Theorem 3.14 in [Nittka 2011], we have

$$
\begin{aligned}
\left|\Gamma-W_{\delta, q}\right|_{L^{s \varepsilon}(\partial M)} \leq \mid-\Delta_{g} & W_{\delta, q}+\left.a(x) W_{\delta, q}\right|_{L^{q+\varepsilon}(M)} \\
+ & \left|f_{0}(\Gamma)+\frac{\partial}{\partial v} W_{\delta, q}+\frac{n-2}{2} b(x) W_{\delta, q}\right|_{L^{(n-1) q /(n-q)+\varepsilon}(\partial M)}
\end{aligned} .
$$

We remark that

$$
q=\frac{2 n+n^{2}\left(\frac{n-2}{n-1}\right) \varepsilon}{n+2+2 n\left(\frac{n-2}{n-1}\right) \varepsilon}=\frac{2 n}{n+2}+O^{+}(\varepsilon) \quad \text { with } 0<O^{+}(\varepsilon)<C \varepsilon
$$

for some positive constant $C$. By direct computation we have

$$
\begin{aligned}
\left|a(x) W_{\delta, q}\right|_{L^{q+\varepsilon}(M)} & \leq C \delta^{2-O^{+}(\varepsilon)}, \\
\left|b(x) W_{\delta, q}\right|_{L^{(n-1) q /(n-q)+\varepsilon}(\partial M)} & \leq C \delta^{1-O^{+}(\varepsilon)} .
\end{aligned}
$$

Moreover, proceeding as in (3-9), (3-10), (3-11) and (3-13) we get

$$
\begin{aligned}
\left|\Delta_{g} W_{\delta, q}\right|_{L^{q+\varepsilon}(M)} & \leq C \delta^{2-O^{+}(\varepsilon)}, \\
\left|f_{0}(\Gamma)+\frac{\partial}{\partial \nu} W_{\delta, q}\right|_{L^{(n-1) q /(n-q)+\varepsilon(\partial M)}} & \leq C \delta^{1-O^{+}(\varepsilon)} .
\end{aligned}
$$

Since $i^{*}\left(f_{\varepsilon}\left(W_{\delta, q}\right)\right)$ solves (1-5), and $i^{*}\left(f_{\varepsilon}|u|^{n /(n-2)+\varepsilon}\left(W_{\delta, q}\right)\right)$ solves (1-5), we again use Theorem 3.14 in [Nittka 2011]. Taking (3-15) and (3-16) into account, 
we finally get

$$
\begin{aligned}
& \left|i^{*}\left(f_{\varepsilon}\left(W_{\delta, q}\right)\right)-i^{*}\left(f_{0}\left(W_{\delta, q}\right)\right)\right|_{L^{s \varepsilon}(\partial M)} \\
& \quad \leq\left|f_{\varepsilon}\left(W_{\delta, q}\right)-f_{0}\left(W_{\delta, q}\right)\right|_{L^{2(n-1) / n+O^{+}(\varepsilon)}(\partial M)} \\
& \leq \delta^{-O^{+}(\varepsilon)}\left(\int _ { \mathbb { R } ^ { n - 1 } } \left[\left(\frac{1}{\delta^{\varepsilon(n-2) / 2}} U^{\varepsilon}(z, 0)-1\right)\right.\right. \\
& \left.\left.\quad \cdot U^{n /(n-2)}(z, 0)\right]^{\frac{2(n-1)}{n}+O^{+}(\varepsilon)} d z\right)^{\frac{1}{2(n-1) / n+O^{+}(\varepsilon)}}+O\left(\delta^{2}\right) \\
& =\delta^{-O^{+}(\varepsilon)}(O(\varepsilon|\ln \delta|)+O(\varepsilon))+O\left(\delta^{2}\right) .
\end{aligned}
$$

Now, choosing $\delta=d \varepsilon$, we can conclude the proof, since

$$
\delta^{-O^{+}(\varepsilon)}=1+O^{+}(\varepsilon)|\ln (\varepsilon d)|=1+O^{+}(\varepsilon|\ln \varepsilon|)=O(1) .
$$

\subsection{Solving (2-8): the remainder term $\phi$.}

Proposition 4. For $a, b \in \mathbb{R}$ with $0<a<b$, there exists a positive constant $C=C(a, b)$ such that, for $\varepsilon$ small, for any $q \in \partial M$ and for any $d \in[a, b]$ there exists a unique $\phi_{\delta, q}$ which solves (2-8). This solution satisfies

$$
\left\|\phi_{\delta, q}\right\|_{\mathcal{H}} \leq C \varepsilon|\ln \varepsilon| .
$$

Moreover the map $q \mapsto \phi_{\delta, q}$ is a $C^{1}(\partial M, \mathcal{H})$ map.

Proof. First of all, we point out that $N$ is a contraction mapping. We remark that the conjugate exponent of $s_{\varepsilon}$ is

$$
s_{\varepsilon}^{\prime}= \begin{cases}\frac{2(n-1)}{n} & \text { in the subcritical case, } \\ \frac{2(n-1)+\varepsilon n(n-2)}{n+\varepsilon n(n-2)} & \text { in the supercritical case. }\end{cases}
$$

By the properties of $i^{*}$ and using the expansion of $f_{\varepsilon}\left(W_{\delta, q}+\phi_{1}\right)$ centered in $W_{\delta, q}+\phi_{2}$ we have

$$
\begin{aligned}
\left\|N\left(\phi_{1}\right)-N\left(\phi_{2}\right)\right\|_{\mathcal{H}} & \leq\left\|f_{\varepsilon}\left(W_{\delta, q}+\phi_{1}\right)-f_{\varepsilon}\left(W_{\delta, q}+\phi_{2}\right)-f_{\varepsilon}^{\prime}\left(W_{\delta, q}\right)\left[\phi_{1}-\phi_{2}\right]\right\|_{L^{s_{\varepsilon}^{\prime}}(\partial M)} \\
& \leq\left\|\left(f_{\varepsilon}^{\prime}\left(W_{\delta, q}+\theta \phi_{1}+(1-\theta) \phi_{2}\right)-f_{\varepsilon}^{\prime}\left(W_{\delta, q}\right)\right)\left[\phi_{1}-\phi_{2}\right]\right\|_{L^{s_{\varepsilon}^{\prime}}(\partial M)}
\end{aligned}
$$

and, since $\left|\phi_{1}-\phi_{2}\right|^{s_{\varepsilon}^{\prime}} \in L^{s_{\varepsilon} / s_{\varepsilon}^{\prime}}(\partial M)$ and $\left|f_{\varepsilon}^{\prime}(\cdot)\right|^{s_{\varepsilon}^{\prime}} \in L^{\left(s_{\varepsilon} / s_{\varepsilon}^{\prime}\right)^{\prime}}(\partial M)$ as $f_{\varepsilon}^{\prime}(\cdot) \in L^{s_{\varepsilon}}(\partial M)$, we have

$$
\begin{aligned}
\| N\left(\phi_{1}\right)- & N\left(\phi_{2}\right) \|_{\mathcal{H}} \\
& \leq\left\|\left(f_{\varepsilon}^{\prime}\left(W_{\delta, q}+\theta \phi_{1}+(1-\theta) \phi_{2}\right)-f_{\varepsilon}^{\prime}\left(W_{\delta, q}\right)\right)\right\|_{L^{s_{\varepsilon}}(\partial M)}\left\|\phi_{1}-\phi_{2}\right\|_{L^{s_{\varepsilon}}(\partial M)} \\
& =\gamma\left\|\phi_{1}-\phi_{2}\right\|_{\mathcal{H}},
\end{aligned}
$$


where

$$
\gamma=\left\|\left(f_{\varepsilon}^{\prime}\left(W_{\delta, q}+\theta \phi_{1}+(1-\theta) \phi_{2}\right)-f_{\varepsilon}^{\prime}\left(W_{\delta, q}\right)\right)\right\|_{L^{s_{\varepsilon}(\partial M)}}<1
$$

provided $\left\|\phi_{1}\right\|_{\mathcal{H}}$ and $\left\|\phi_{2}\right\|_{\mathcal{H}}$ are sufficiently small.

In the same way we can prove that $\|N(\phi)\|_{\mathcal{H}} \leq \gamma\|\phi\|_{\mathcal{H}}$ with $\gamma<1$ if $\|\phi\|_{\mathcal{H}}$ is sufficiently small.

Next, by Lemmas 2 and 3 we have

$$
\left\|L^{-1}\left(N(\phi)+R_{\varepsilon, \delta, q}\right)\right\|_{\mathcal{H}} \leq C\left(\gamma\|\phi\|_{\mathcal{H}}+\varepsilon|\ln \varepsilon|\right),
$$

where $C=\max \left\{C_{0}, C_{0} C_{1}\right\}>0$, for the constants $C_{0}, C_{1}$ which appear in Lemmas 2 and 3. Notice that, given $C>0$, it is possible (up to a choice of $\|\phi\|_{\mathcal{H}}$ sufficiently small) to choose $0<C \gamma<\frac{1}{2}$.

Now, if $\|\phi\|_{\mathcal{H}} \leq 2 C \varepsilon|\ln \varepsilon|$, then the map

$$
T(\phi):=L^{-1}\left(N(\phi)+R_{\varepsilon, \delta, q}\right)
$$

is a contraction from the ball $\|\phi\|_{\mathcal{H}} \leq 2 C \varepsilon|\ln \varepsilon|$ in itself, so, by the fixed point theorem, there exists a unique $\phi_{\delta, q}$ with $\left\|\phi_{\delta, q}\right\|_{\mathcal{H}} \leq 2 C \varepsilon|\ln \varepsilon|$ solving (3-1), and hence (2-8). The regularity of the map $q \mapsto \phi_{\delta, q}$ can be proven via the implicit function theorem.

\section{The reduced problem}

Problem (1-5) has a variational structure. Weak solutions to (1-5) are critical points of the energy functional $J_{\varepsilon}: \mathcal{H} \rightarrow \mathbb{R}$ given by

$$
\begin{aligned}
J_{\varepsilon}(u)=\frac{1}{2} \int_{M}\left(|\nabla u|^{2}+a(x) u^{2}\right) d \mu_{g} & \\
& +\frac{n-2}{4} \int_{\partial M} b(x) u^{2} d \sigma-\frac{(n-2)^{2}}{2 n-2 \pm \varepsilon(n-2)} \int_{\partial M} u^{(2 n-2) /(n-2) \pm \varepsilon} d \sigma .
\end{aligned}
$$

Let us introduce the reduced energy $I_{\varepsilon}:(0,+\infty) \times \partial M \rightarrow \mathbb{R}$ by

$$
I_{\varepsilon}(d, q):=J_{\varepsilon}\left(W_{\varepsilon d, q}+\phi_{\varepsilon d, q}\right),
$$

where the remainder term $\phi_{\varepsilon d, q}$ has been found in Proposition 4.

4.1. The reduced energy. Here we use the following expansion for the metric tensor on $M$ :

$$
\begin{aligned}
& g^{i j}(y)=\delta_{i j}+2 h_{i j}(0) y_{n}+O\left(|y|^{2}\right) \text { for } i, j=1, \ldots, n-1, \\
& g^{i n}(y)=\delta_{i n} \quad \text { for } i=1, \ldots, n-1, \\
& \sqrt{g}(y)=1-(n-1) H(0) y_{n}+O\left(|y|^{2}\right),
\end{aligned}
$$


where $\left(y_{1}, \ldots, y_{n}\right)$ are the Fermi coordinates and, by definition of $h_{i j}$,

$$
H=\frac{1}{n-1} \sum_{i}^{n-1} h_{i i} .
$$

We also recall that on $\partial M$ the Fermi coordinates coincide with the exponential ones, so we have that

$$
\sqrt{g}\left(y_{1}, \ldots, y_{n-1}, 0\right)=1+O\left(|y|^{2}\right) .
$$

To improve the readability of this paper, hereafter we write $z=\left(z_{1}, \ldots, z_{n-1}\right)$ to indicate the first $n-1$ Fermi coordinates and $t$ to indicate the last one, so $\left(y_{1}, \ldots, y_{n-1}, y_{n}\right)=(z, t)$. Moreover, indices $i, j$ conventionally refer to sums from 1 to $n-1$, while $l, m$ usually refer to sums from 1 to $n$.

Proposition 5. (i) If $\left(d_{0}, q_{0}\right) \in(0,+\infty) \times \partial M$ is a critical point for the reduced energy $I_{\mathcal{E}}$ defined in (4-1), then $W_{\varepsilon d_{0}, q_{0}}+\phi_{\varepsilon d_{0}, q_{0}} \in \mathcal{H}$ solves problem (1-5).

(ii) It holds true that

$$
\left\{\begin{array}{l}
I_{\varepsilon}(d, q)=c_{n}(\varepsilon)+\varepsilon\left[\alpha_{n} d \varphi(q)-\beta_{n} \ln d\right]+o(\varepsilon) \quad \text { in the subcritical case, } \\
I_{\varepsilon}(d, q)=c_{n}(\varepsilon)+\varepsilon\left[\alpha_{n} d \varphi(q)+\beta_{n} \ln d\right]+o(\varepsilon) \quad \text { in the supercritical case }
\end{array}\right.
$$

$C^{0}$-uniformly with respect to $d$ in compact subsets of $(0,+\infty)$ and $q \in \partial M$. Here $c_{n}(\varepsilon)$ is a constant which only depends on $\varepsilon$ and $n, \alpha_{n}$ and $\beta_{n}$ are positive constants which only depend on $n$, and $\varphi(q)=h(q)-H_{g}(q)$ is the function defined in (1-4) .

Proof. (i) Set $q:=q(y)=\psi_{q_{0}}^{\partial}(y)$. Since $\left(d_{0}, q_{0}\right)$ is a critical point, we have, for any $h \in 1, \ldots, n-1$,

$$
\begin{aligned}
0 & =\left.\frac{\partial}{\partial y_{h}} I_{\varepsilon}\left(d, \psi_{q_{0}}^{\partial}(y)\right)\right|_{y=0} \\
& =\left\langle\left\langle W_{\varepsilon d, q(y)}+\phi_{\varepsilon d, q(y)}-i^{*}\left(f_{\varepsilon}\left(W_{\varepsilon d, q(y)}+\phi_{\varepsilon d, q(y)}\right),\right.\right.\right. \\
& \left.\left.=\sum_{i=0} \frac{\partial}{\partial y_{h}} W_{\varepsilon d, q(y)}+\frac{\partial}{\partial y_{h}} \phi_{\varepsilon d, q(y)}\right\rangle\right\rangle\left._{H}\right|_{y=0}\left\langle\left.\left.\left\langle Z_{\varepsilon d, q(y)}^{i}, \frac{\partial}{\partial y_{h}} W_{\varepsilon d, q(y)}+\frac{\partial}{\partial y_{h}} \phi_{\varepsilon d, q(y)}\right\rangle\right|_{H}\right|_{y=0}\right. \\
& =\sum_{i=0}^{n-1} c_{\varepsilon}^{i}\left\langle\left\langle Z_{\varepsilon d, q(y)}^{i}, \frac{\partial}{\partial y_{h}} W_{\varepsilon d, q(y)} \|\left.\left.\right|_{H}\right|_{y=0}-\left.\sum_{i=0}^{n-1} c_{l}^{i}\left\langle\left\langle\frac{\partial}{\partial y_{h}} Z_{\varepsilon d, q(y)}^{i}, \phi_{\varepsilon d, q(y)}\right\rangle\right\rangle_{H}\right|_{y=0},\right.\right.
\end{aligned}
$$

using that $\phi_{\varepsilon d, q(y)}$ is a solution of (2-8) and that

$$
\left\langle\left\langle Z_{\varepsilon d, q(y)}^{i}, \frac{\partial}{\partial y_{h}} \phi_{\varepsilon d, q(y)}\right\rangle\right\rangle_{H}=-\left\langle\left\langle\frac{\partial}{\partial y_{h}} Z_{\varepsilon d, q(y)}^{i}, \phi_{\varepsilon d, q(y)}\right\rangle_{H}\right.
$$


since $\phi_{\varepsilon d, q(y)} \in K_{\varepsilon d, q(y)}^{\perp}$ for all $y$. Now it is enough to observe that

$$
\begin{aligned}
& \left\langle\left\langle\frac{\partial}{\partial y_{h}} Z_{\varepsilon d, q(y)}^{i}, \phi_{\varepsilon d, q(y)}\right\rangle_{H} \leq\left\|\frac{\partial}{\partial y_{h}} Z_{\varepsilon d, q(y)}^{i}\right\|_{H}\left\|\phi_{\varepsilon d, q(y)}\right\|_{H}=o(1),\right. \\
& \left\langle\left\langle Z_{\varepsilon d, q(y)}^{i}, \frac{\partial}{\partial y_{h}} W_{\varepsilon d, q(y)}\right\rangle_{H}=\frac{1}{\varepsilon d} \|\left\langle Z_{\varepsilon d, q(y)}^{i}, Z_{\varepsilon d, q(y)}^{h}\right\rangle_{H}=\frac{1}{\varepsilon d} \delta^{i h}+o(1),\right.
\end{aligned}
$$

to conclude that

$$
0=\frac{1}{\varepsilon d} \sum_{i=0}^{n-1} c_{\varepsilon}^{i}\left(\delta^{i h}+o(1)\right),
$$

and so $c_{\varepsilon}^{i}=0$ for all $i=0, \ldots, n-1$. This concludes the proof of (i).

(ii) We prove (ii) in two steps.

Step 1. We prove that for $\varepsilon$ small enough and for any $q \in \partial M$,

$$
\left|J_{\varepsilon}\left(W_{\delta, q}+\phi_{\delta, q}\right)-J_{\varepsilon}\left(W_{\delta, q}\right)\right| \leq\left\|\phi_{\delta, q}\right\|_{\mathcal{H}}^{2}+C \varepsilon|\ln \varepsilon|\left\|\phi_{\delta, q}\right\|_{\mathcal{H}}=o(\varepsilon) .
$$

We have

$$
\begin{aligned}
\left|J_{\varepsilon}\left(W_{\delta, q}+\phi_{\delta, q}\right)-J_{\varepsilon}\left(W_{\delta, q}\right)\right| & \\
= & \left|\int_{M}\left[-\Delta_{g} W_{\delta, q}+a(x) W_{\delta, q}\right] \phi_{\delta, q} d \mu_{g}\right|+\frac{1}{2}\left\|\phi_{\delta, q}\right\|_{H}^{2} \\
& +\left|\int_{\partial M}\left[\frac{\partial}{\partial v} W_{\delta, q}+\frac{n-2}{2} b(x) W_{\delta, q}-f_{0}\left(W_{\delta, q}\right)\right] \phi_{\delta, q} d \sigma\right| \\
& +\left|\int_{\partial M}\left[f_{0}\left(W_{\delta, q}\right)-f_{\varepsilon}\left(W_{\delta, q}\right)\right] \phi_{\delta, q} d \sigma\right| \\
& +\mid \int_{\partial M} \frac{(n-2)^{2}}{2 n-2 \pm \varepsilon(n-2)}\left[\left(W_{\delta, q}+\phi_{\delta, q}\right)^{(2 n-2) /(n-2) \pm \varepsilon}-W_{\delta, q}^{(2 n-2) /(n-2) \pm \varepsilon}\right] \\
& -f_{\varepsilon}\left(W_{\delta, q}\right) \phi_{\delta, q} d \sigma \mid .
\end{aligned}
$$

With the same estimate of $I_{1}$ in Lemma 3 we obtain that

$$
\left|\int_{M}\left[-\Delta_{g} W_{\delta, q}+a(x) W_{\delta, q}\right] \phi_{\delta, q} d \mu_{g}\right|=O(\delta)\left\|\phi_{\delta, q}\right\|_{H},
$$

and in light of the estimate of $I_{2}$ and $I_{3}$ in Lemma 3 we get

$$
\left|\int_{\partial M}\left[\frac{\partial}{\partial v} W_{\delta, q}+\frac{n-2}{2} b(x) W_{\delta, q}-f_{0}\left(W_{\delta, q}\right)\right] \phi_{\delta, q} d \sigma\right|=O(\delta)\left\|\phi_{\delta, q}\right\|_{H} .
$$


In the subcritical case, following the computation in (3-17) we obtain

$$
\begin{aligned}
& \left|\int_{\partial M}\left[f_{0}\left(W_{\delta, q}\right)-f_{\varepsilon}\left(W_{\delta, q}\right)\right] \phi_{\delta, q} d \sigma\right| \\
& \leq C\left|f_{0}\left(W_{\delta, q}\right)-f_{\varepsilon}\left(W_{\delta, q}\right)\right|_{L^{2(n-1) / n}(\partial M)}\left|\phi_{\delta, q}\right|_{L^{2(n-1) /(n-2)}(\partial M)} \\
& \quad=[O(\varepsilon)+O(\varepsilon \ln \delta)]\left\|\phi_{\delta, q}\right\|_{H}=O(\varepsilon|\ln \varepsilon|)\left\|\phi_{\delta, q}\right\|_{H},
\end{aligned}
$$

and in a similar way, for the supercritical case, in light of (3-18) we get

$$
\begin{aligned}
& \mid \int_{\partial M}\left[f_{0}(\right.\left.\left.W_{\delta, q}\right)-f_{\varepsilon}\left(W_{\delta, q}\right)\right] \phi_{\delta, q} d \sigma \mid \\
& \leq C\left|f_{0}\left(W_{\delta, q}\right)-f_{\varepsilon}\left(W_{\delta, q}\right)\right|_{L^{2(n-1) / n+O^{+}(\varepsilon)}(\partial M)}\left|\phi_{\delta, q}\right|_{L^{2(n-1) /(n-2)-O O^{+}(\varepsilon)}(\partial M)} \\
& \leq\left(\delta^{-O^{+}(\varepsilon)}(O(\varepsilon \ln \delta)+O(\varepsilon))+O\left(\delta^{2}\right)\right)\left\|\phi_{\delta, q}\right\|_{H}=O(\varepsilon|\ln \varepsilon|)\left\|\phi_{\delta, q}\right\|_{H} .
\end{aligned}
$$

Finally, by the Taylor expansion formula, for some $\theta \in(0,1)$ we immediately have

$$
\begin{gathered}
\left|\int_{\partial M} \frac{(n-2)^{2}}{2 n-2 \pm \varepsilon(n-2)}\left[\left(W_{\delta, q}+\phi_{\delta, q}\right)^{\frac{2 n-2}{n-2} \pm \varepsilon}-W_{\delta, q}^{\frac{2 n-2}{n-2} \pm \varepsilon}\right]-f_{\varepsilon}\left(W_{\delta, q}\right) \phi_{\delta, q} d \sigma\right| \\
=\left|\frac{n \pm \varepsilon(n-2)}{2} \int_{\partial M}\left(W_{\delta, q}+\theta \phi_{\delta, q}\right)^{\frac{2}{n-2} \pm \varepsilon} \phi_{\delta, q}^{2} d \sigma\right| \\
\leq C\left[\int_{\partial M}\left|W_{\delta, q}+\theta \phi_{\delta, q}\right|\left(\frac{2}{n-2} \pm \varepsilon\right) \frac{s_{\varepsilon}}{s_{\varepsilon}-2} d \sigma\right]^{\frac{s_{\varepsilon}-2}{S_{\varepsilon}}}\left[\int_{\partial M}\left|\phi_{\delta, q}\right|^{s_{\varepsilon}} d \sigma\right]^{\frac{2}{s_{\varepsilon}}} \\
\leq C\left|W_{\delta, q}+\theta \phi_{\delta, q}\right|_{L^{s_{\varepsilon}}}^{s_{\varepsilon}-2}\left\|\phi_{\delta, q}\right\|_{\mathcal{H}}^{2} \leq C\left\|\phi_{\delta, q}\right\|_{\mathcal{H}}^{2} .
\end{gathered}
$$

Choosing $\delta=d \varepsilon$, and recalling that, by Proposition 4, $\left\|\phi_{\delta, q}\right\|_{\mathcal{H}}=O(\varepsilon|\ln \varepsilon|)$ concludes the proof.

Step 2. We prove that

$$
\begin{aligned}
& J_{\varepsilon}\left(W_{\delta, q}\right) \\
& \quad=C(\varepsilon)+\varepsilon\left(d \frac{n-2}{4}[b(q)-H(q)] \pm \ln d \frac{(n-2)^{3}(n-3)}{4(n-2)(2 n-2)}\right) \omega_{n-1} I_{n-2}^{n-2}+o(\varepsilon)
\end{aligned}
$$

$C^{0}$-uniformly with respect to $d$ in compact subsets of $(0,+\infty)$ and $q \in \partial M$, where

$$
\begin{aligned}
& C(\varepsilon)=\frac{1}{2} \int_{\mathbb{R}_{+}^{n}}|\nabla U(y)|^{2} d y \\
&-\frac{(n-2)^{2}}{2 n-2} \int_{\mathbb{R}^{n-1}} U^{\frac{2 n-2}{n-2}}(z, 0) d z \pm \varepsilon \frac{(n-2)^{3}}{2 n-2} \int_{\mathbb{R}^{n-1}} U^{\frac{2 n-2}{n-2}}(z, 0) d z \\
& \mp \varepsilon \frac{(n-2)^{2}}{2 n-2} \int_{\mathbb{R}^{n-1}} U^{\frac{2 n-2}{n-2}}(z, 0) \ln U(z, 0) d z \\
& \mp \varepsilon|\ln \varepsilon| \frac{(n-2)^{3}}{2(2 n-2)} \int_{\mathbb{R}^{n-1}} U^{\frac{2 n-2}{n-2}}(z, 0) d z,
\end{aligned}
$$


and

$$
I_{n-2}^{n-2}=\int_{0}^{\infty} \frac{s^{n-2}}{\left(1+s^{2}\right)^{n-2}} d z
$$

and $\omega_{n-1}$ is the volume of the $(n-1)$-dimensional unit ball.

We compute each term separately. First, we have, by a change of variables and by (4-2), (4-3) and (4-4),

$$
\begin{array}{r}
\int_{M}\left|\nabla W_{\delta, q}\right|^{2} d \mu_{g}= \\
\sum_{l, m=1}^{n} \int_{\mathbb{R}_{+}^{n}} g^{l m}(\delta y) \frac{\partial}{\partial y_{l}} U(y) \frac{\partial}{\partial y_{m}} U(y) \sqrt{g}(\delta y) d y+o(\delta) \\
=\int_{\mathbb{R}_{+}^{n}}|\nabla U(y)|^{2} d y-\delta(n-1) H(q) \int_{\mathbb{R}_{+}^{n}} y_{n}|\nabla U(y)|^{2} d y \\
+2 \delta \sum_{i, j=1}^{n-1} \int_{\mathbb{R}_{+}^{n}} y_{n} h_{i j}(q) \frac{\partial}{\partial y_{i}} U(y) \frac{\partial}{\partial y_{j}} U(y) d y+o(\delta) .
\end{array}
$$

By a symmetry argument we can simplify the last integral to obtain, in a more compact form,

$$
\begin{aligned}
& \frac{1}{2} \int_{M}\left|\nabla W_{\delta, q}\right|^{2} d \mu_{g}=\frac{1}{2} \int_{\mathbb{R}_{+}^{n}}|\nabla U|^{2}-\delta \frac{(n-1) H(q)}{2} \int_{\mathbb{R}_{+}^{n}} y_{n}|\nabla U|^{2} \\
& +\delta \sum_{i=1}^{n-1} h_{i i}(q) \int_{\mathbb{R}_{+}^{n}} y_{n}\left(\frac{\partial U}{\partial y_{i}}(y)\right)^{2}+o(\delta) .
\end{aligned}
$$

Since $\frac{\partial U}{\partial y_{i}}=\frac{\partial U}{\partial y_{l}}$ for all $i, l=1, \ldots, n-1$, by (4-9) we get

$$
\begin{aligned}
\sum_{i=1}^{n-1} h_{i i}(q) \int_{\mathbb{R}_{+}^{n}} y_{n}\left(\frac{\partial U}{\partial y_{i}}(y)\right)^{2} d y & =\frac{1}{n-1} \sum_{i=1}^{n-1} h_{i i}(q) \int_{\mathbb{R}_{+}^{n}} y_{n} \sum_{l=1}^{n-1}\left(\frac{\partial U}{\partial y_{l}}(y)\right)^{2} d y \\
& =\frac{H(q)}{4} \int_{\mathbb{R}^{n-1}} U^{2}(z, 0) d z,
\end{aligned}
$$

and in light of (4-7) we conclude that

$$
\frac{1}{2} \int_{M}\left|\nabla W_{\delta, q}\right|^{2} d \mu_{g}=\frac{1}{2} \int_{\mathbb{R}_{+}^{n}}|\nabla U|^{2}-\delta \frac{(n-2) H(q)}{4} \int_{\mathbb{R}^{n-1}} U^{2}(z, 0) d z+o(\delta) .
$$

By a change of variables, we immediately obtain

$$
\frac{1}{2} \int_{M} a(x)\left|W_{\delta, q}\right|^{2} d \mu_{g}=\frac{\delta^{2}}{2} \int_{\mathbb{R}_{+}^{n}} a(x) U^{2}(y) \sqrt{g}(\delta y) d y+o\left(\delta^{2}\right)=O\left(\delta^{2}\right) .
$$


Coming to the boundary integral, we get, by a change of variables, by (4-6), and by expanding $b$,

$$
\begin{aligned}
\frac{n-2}{4} \int_{\partial M} b(z)\left|W_{\delta, q}\right|^{2} d \sigma & =\delta \frac{n-2}{4} \int_{\mathbb{R}^{n-1}} b(\delta z) U^{2}(z, 0) \sqrt{g}(\delta z) d z+O\left(\delta^{2}\right) \\
& =\delta b(q) \frac{n-2}{4} \int_{\mathbb{R}^{n-1}} U^{2}(z, 0) d z+O\left(\delta^{2}\right) .
\end{aligned}
$$

Introducing the abbreviation $U_{n}(z)=U^{(2 n-2) /(n-2)}(z, 0)$, by (3-15), (3-16) and (4-6), we have

$$
\begin{aligned}
\int_{\partial M}\left|W_{\delta, q}\right|^{(2 n-2) /(n-2) \pm \varepsilon} d \sigma & \\
= & \int_{\mathbb{R}^{n-1}} \delta^{\mp \varepsilon(n-2) / 2} U_{n}(z) U^{ \pm \varepsilon}(z, 0) \sqrt{g}(\delta z) d z+o(\delta) \\
= & \int_{\mathbb{R}^{n-1}} U_{n}(z) d z \pm \varepsilon \int_{\mathbb{R}^{n-1}} U_{n}(z) \ln U(z, 0) d z \mp \frac{n-2}{2} \varepsilon \ln \delta \int_{\mathbb{R}^{n-1}} U_{n}(z) d z \\
& +o(\delta)+O\left(\varepsilon^{2}\right)+O\left(\varepsilon^{2} \ln \delta\right),
\end{aligned}
$$

and, since $\frac{(n-2)^{2}}{2 n-2 \pm \varepsilon(n-2)}=\frac{(n-2)^{2}}{2 n-2} \mp \varepsilon \frac{(n-2)^{3}}{2 n-2}$, we get

$$
\begin{aligned}
&-\frac{(n-2)^{2}}{2 n-2 \pm \varepsilon(n-2)} \int_{\partial M}\left|W_{\delta, q}\right|^{(2 n-2) /(n-2)-\varepsilon} d \sigma \\
&=-\frac{(n-2)^{2}}{2 n-2} \int_{\mathbb{R}^{n-1}} U_{n}(z) d z \pm \varepsilon \frac{(n-2)^{3}}{2 n-2} \int_{\mathbb{R}^{n-1}} U_{n}(z) d z \\
& \mp \varepsilon \frac{(n-2)^{2}}{2 n-2} \int_{\mathbb{R}^{n-1}} U_{n}(z) \ln U(z, 0) d z \pm \frac{(n-2)^{3}}{2(2 n-2)} \varepsilon \ln \delta \int_{\mathbb{R}^{n-1}} U_{n}(z) d z \\
&+o(\delta)+O\left(\varepsilon^{2}\right)+O\left(\varepsilon^{2} \ln \delta\right) .
\end{aligned}
$$

Notice that, with the choice $\delta=d \varepsilon$ it holds that $o(\delta)+O\left(\varepsilon^{2}\right)+O\left(\varepsilon^{2} \ln \delta\right)=o(\varepsilon)$ and $\varepsilon \ln \delta=\varepsilon \ln d-\varepsilon|\ln \varepsilon|$. At this point we have

$$
\begin{aligned}
J_{\varepsilon}\left(W_{\delta, q}\right)=C(\varepsilon)+\varepsilon d \frac{n-2}{4}[b(q)- & H(q)] \int_{\mathbb{R}^{n-1}} U^{2}(z, 0) d z \\
& \pm \varepsilon \frac{(n-2)^{3}}{2(2 n-2)} \ln d \int_{\mathbb{R}^{n-1}} U_{n}(z) d z+o(\varepsilon|\ln \varepsilon|) .
\end{aligned}
$$

To conclude, observe that

$$
\int_{\mathbb{R}^{n-1}} U^{2}(z, 0) d z=\omega_{n-1} I_{n-2}^{n-2} \quad \text { and } \quad \int_{\mathbb{R}^{n-1}} U_{n}(z) d z=\omega_{n-1} I_{n-1}^{n-2},
$$


where

$$
I_{\beta}^{\alpha}=\int_{0}^{\infty} \frac{s^{\alpha}}{\left(1+s^{2}\right)^{\beta}} d s .
$$

The conclusion follows after we observe that $I_{n-1}^{n-2}=\frac{n-3}{2(n-2)} I_{n-2}^{n-2}$ (for a proof, see [Almaraz 2011b, Lemma 9.4(b)]).

4.2. Proof of Theorem 1. Let us introduce

$$
\hat{I}(d, q)=\alpha_{n} d \varphi(q)-\beta_{n} \ln d .
$$

If $q_{0}$ is a local minimizer of $\varphi(q)$ with $\varphi\left(q_{0}\right)>0$, set $d_{0}=\beta_{n} /\left(\alpha_{n} \varphi\left(q_{0}\right)\right)>0$. Thus the pair $\left(d_{0}, q_{0}\right)$ is a critical point for $\hat{I}$. Moreover, since there exists a neighborhood $B$ such that $\varphi(q)>\varphi\left(q_{0}\right)$ on $\partial B$, it is possible to find a neighborhood $\widetilde{B} \subset[a, b] \times \partial M,\left(d_{0}, q_{0}\right) \in \widetilde{B}$ such that $\hat{I}(d, q)>\hat{I}\left(d_{0}, q_{0}\right)$ for $(d, q) \in \partial \widetilde{B}$. Since, in the subcritical case, by (i) of Proposition 5 we have

$$
I_{\varepsilon}(d, q)=c_{n}(\varepsilon)+\varepsilon \hat{I}(d, q)+o(\varepsilon),
$$

we get that for $\varepsilon$ sufficiently small there is a $\left(d^{*}, q^{*}\right) \in \widetilde{B}$ such that $W_{\varepsilon d^{*}, q^{*}}+\phi_{\varepsilon d^{*}, q^{*}}$ is a critical point for $I_{\varepsilon}$. Then, by (i) of Proposition $5, W_{\varepsilon d^{*}, q^{*}}+\phi_{\varepsilon d^{*}, q^{*}} \in \mathcal{H}$ is a solution for problem (1-5) in the subcritical case.

The proof for the supercritical case follows in a similar way.

4.3. Some technicalities. If $U$ is a solution of (2-5), then the following hold:

$$
\begin{aligned}
\int_{\mathbb{R}_{+}^{n}} t|\nabla U|^{2} d z d t & =\frac{1}{2} \int_{\mathbb{R}^{n-1}} U^{2}(z, 0) d z, \\
\int_{\mathbb{R}_{+}^{n}} t|\nabla U|^{2} d z d t & =2 \int_{\mathbb{R}_{+}^{n}} t\left|\partial_{t} U\right|^{2} d z d t, \\
\int_{\mathbb{R}_{+}^{n}} t \sum_{i=1}^{n-1}\left|\partial_{z_{i}} U\right|^{2} d z d t & =\frac{1}{4} \int_{\mathbb{R}^{n-1}} U^{2}(z, 0) d z .
\end{aligned}
$$

Proof. To simplify the notation, we set

$$
\eta=(z, t) \in \mathbb{R}_{+}^{n} \quad \text { where } z \in \mathbb{R}^{n-1} \text { and } t \geq 0 .
$$

The first estimate can be obtained by integration by parts, taking into account that $\Delta U=0$. Indeed,

$$
\begin{aligned}
\int_{\mathbb{R}_{+}^{n}} \eta_{n}|\nabla U|^{2} \delta \eta & =-\sum_{l=1}^{n} \int_{\mathbb{R}_{+}^{n}} U \partial_{l}\left[\eta_{n} \partial_{l} U\right] \delta \eta=-\int_{\mathbb{R}_{+}^{n}} U \partial_{n} U \delta \eta-\int_{\mathbb{R}_{+}^{n}} \eta_{n} U \Delta U \delta \eta \\
& =-\frac{1}{2} \int_{\mathbb{R}_{+}^{n}} \partial_{n}\left[U^{2}\right] \delta \eta=\frac{1}{2} \int_{\mathbb{R}^{n-1}} U^{2}(z, 0) d z .
\end{aligned}
$$


To obtain (4-8), we proceed in a similar way: since $\Delta U=0$ we have

$$
\begin{aligned}
0 & =-\int_{\mathbb{R}_{+}^{n}} \Delta U \eta_{n}^{2} \partial_{n} U \delta \eta=\sum_{l=1}^{n} \int_{\mathbb{R}_{+}^{n}} \partial_{l} U \partial_{l}\left[\eta_{n}^{2} \partial_{n} U\right] \delta \eta \\
& =\int_{\mathbb{R}_{+}^{n}} 2 \eta_{n}\left|\partial_{n} U\right|^{2} \delta \eta+\sum_{l=1}^{n} \int_{\mathbb{R}_{+}^{n}} \eta_{n}^{2} \partial_{l} U \partial_{l n}^{2} U \delta \eta \\
& =\int_{\mathbb{R}_{+}^{n}} 2 \eta_{n}\left|\partial_{n} U\right|^{2} \delta \eta+\frac{1}{2} \int_{\mathbb{R}_{+}^{n}} \eta_{n}^{2} \partial_{n}|\nabla U|^{2} \delta \eta \\
& =\int_{\mathbb{R}_{+}^{n}} 2 \eta_{n}\left|\partial_{t} U\right|^{2} \delta \eta-\int_{\mathbb{R}_{+}^{n}} \eta_{n}|\nabla U|^{2} \delta \eta,
\end{aligned}
$$

so (4-8) is proved. Now (4-9) is a direct consequence of the first two equalities. In fact, by (4-8) we have

$$
\begin{aligned}
\int_{\mathbb{R}_{+}^{n}} \eta_{n}|\nabla U|^{2} \delta \eta & =\int_{\mathbb{R}_{+}^{n}} \eta_{n} \sum_{i=1}^{n-1}\left|\partial_{i} U\right|^{2} \delta \eta+\int_{\mathbb{R}_{+}^{n}} \eta_{n}\left|\partial_{n} U\right|^{2} \delta \eta \\
& =\int_{\mathbb{R}_{+}^{n}} \eta_{n} \sum_{i=1}^{n-1}\left|\partial_{i} U\right|^{2} \delta \eta+\frac{1}{2} \int_{\mathbb{R}_{+}^{n}} \eta_{n}|\nabla U|^{2} \delta \eta .
\end{aligned}
$$

Thus,

$$
\int_{\mathbb{R}_{+}^{n}} \eta_{n} \sum_{i=1}^{n-1}\left|\partial_{i} U\right|^{2} \delta \eta=\frac{1}{2} \int_{\mathbb{R}_{+}^{n}} \eta_{n}|\nabla U|^{2} \delta \eta,
$$

and in light of (4-7) we get the proof.

\section{References}

[Almaraz 2010] S. d. M. Almaraz, "An existence theorem of conformal scalar-flat metrics on manifolds with boundary”, Pacific J. Math. 248:1 (2010), 1-22. MR Zbl

[Almaraz 2011a] S. d. M. Almaraz, "Blow-up phenomena for scalar-flat metrics on manifolds with boundary", J. Differential Equations 251:7 (2011), 1813-1840. MR Zbl

[Almaraz 2011b] S. d. M. Almaraz, "A compactness theorem for scalar-flat metrics on manifolds with boundary", Calc. Var. Partial Differential Equations 41:3-4 (2011), 341-386. MR Zbl

[Aubin 1976] T. Aubin, "Équations différentielles non linéaires et problème de Yamabe concernant la courbure scalaire", J. Math. Pures Appl. (9) 55:3 (1976), 269-296. MR Zbl

[Brendle 2008] S. Brendle, "Blow-up phenomena for the Yamabe equation", J. Amer. Math. Soc. 21:4 (2008), 951-979. MR Zbl

[Brendle and Chen 2014] S. Brendle and S.-Y. S. Chen, "An existence theorem for the Yamabe problem on manifolds with boundary”, J. Eur. Math. Soc. (JEMS) 16:5 (2014), 991-1016. MR Zbl

[Brendle and Marques 2009] S. Brendle and F. C. Marques, "Blow-up phenomena for the Yamabe equation, II", J. Differential Geom. 81:2 (2009), 225-250. MR Zbl 
[Cherrier 1984] P. Cherrier, "Problèmes de Neumann non linéaires sur les variétés riemanniennes", $J$. Funct. Anal. 57:2 (1984), 154-206. MR Zbl

[Druet 2003] O. Druet, "From one bubble to several bubbles: The low-dimensional case", J. Differential Geom. 63:3 (2003), 399-473. MR Zbl

[Druet 2004] O. Druet, "Compactness for Yamabe metrics in low dimensions", Int. Math. Res. Not. 2004:23 (2004), 1143-1191. MR Zbl

[Druet and Hebey 2005a] O. Druet and E. Hebey, "Blow-up examples for second order elliptic PDEs of critical Sobolev growth”, Trans. Amer. Math. Soc. 357:5 (2005), 1915-1929. MR Zbl

[Druet and Hebey 2005b] O. Druet and E. Hebey, "Elliptic equations of Yamabe type", Int. Math. Res. Surv. 1 (2005), 1-113. MR Zbl

[Escobar 1992a] J. F. Escobar, "Conformal deformation of a Riemannian metric to a scalar flat metric with constant mean curvature on the boundary", Ann. of Math. (2) 136:1 (1992), 1-50. MR Zbl

[Escobar 1992b] J. F. Escobar, "The Yamabe problem on manifolds with boundary", J. Differential Geom. 35:1 (1992), 21-84. MR Zbl

[Esposito and Pistoia 2014] P. Esposito and A. Pistoia, "Blowing-up solutions for the Yamabe equation", Port. Math. 71:3-4 (2014), 249-276. MR Zbl

[Esposito et al. 2014] P. Esposito, A. Pistoia, and J. Vétois, "The effect of linear perturbations on the Yamabe problem", Math. Ann. 358:1-2 (2014), 511-560. MR Zbl

[Felli and Ould Ahmedou 2003] V. Felli and M. Ould Ahmedou, "Compactness results in conformal deformations of Riemannian metrics on manifolds with boundaries", Math. Z. 244:1 (2003), 175-210. MR Zbl

[Felli and Ould Ahmedou 2005] V. Felli and M. Ould Ahmedou, "A geometric equation with critical nonlinearity on the boundary", Pacific J. Math. 218:1 (2005), 75-99. MR Zbl

[Han and Li 1999] Z.-C. Han and Y. Li, "The Yamabe problem on manifolds with boundary: Existence and compactness results”, Duke Math. J. 99:3 (1999), 489-542. MR Zbl

[Khuri et al. 2009] M. A. Khuri, F. C. Marques, and R. M. Schoen, "A compactness theorem for the Yamabe problem”, J. Differential Geom. 81:1 (2009), 143-196. MR Zbl

[Marques 2005] F. C. Marques, "Existence results for the Yamabe problem on manifolds with boundary", Indiana Univ. Math. J. 54:6 (2005), 1599-1620. MR Zbl

[Marques 2007] F. C. Marques, "Conformal deformations to scalar-flat metrics with constant mean curvature on the boundary”, Comm. Anal. Geom. 15:2 (2007), 381-405. MR Zbl

[Micheletti et al. 2009] A. M. Micheletti, A. Pistoia, and J. Vétois, "Blow-up solutions for asymptotically critical elliptic equations on Riemannian manifolds", Indiana Univ. Math. J. 58:4 (2009), 1719-1746. MR Zbl

[Nittka 2011] R. Nittka, "Regularity of solutions of linear second order elliptic and parabolic boundary value problems on Lipschitz domains”, J. Differential Equations 251:4-5 (2011), 860-880. MR Zbl

[Schoen 1984] R. Schoen, "Conformal deformation of a Riemannian metric to constant scalar curvature”, J. Differential Geom. 20:2 (1984), 479-495. MR Zbl

[Trudinger 1968] N. S. Trudinger, "Remarks concerning the conformal deformation of Riemannian structures on compact manifolds", Ann. Scuola Norm. Sup. Pisa (3) 22 (1968), 265-274. MR Zbl

[Yamabe 1960] H. Yamabe, "On a deformation of Riemannian structures on compact manifolds", Osaka Math. J. 12 (1960), 21-37. MR Zbl 
Received May 29, 2015. Revised January 17, 2016.

MARCO GHIMENTI

DiPARTIMENTO DI MATEMATICA

UNIVERSITÀ DI PISA

VIA F. BUONARROTI 1/C

56127 PISA

ITALY

marco.ghimenti@dma.unipi.it

Anna Maria Micheletti

DiPARTIMENTO Di MATEMATICA

UNIVERSITÀ DI PISA

VIA F. BUONARROTI $1 / \mathrm{C}$

56127 PISA

ITALY

a.micheletti@dma.unipi.it

Angela Pistoia

DipARTIMENTO SCIENZE di BASE E APPLICATE PER L'INGEgNERIA

UNIVERSTÀ DI ROMA "LA SAPIENZA"

VIA ANTONIO SCARPA 16

00161 RoMA

ITALY

angela.pistoia@uniroma1.it 


\title{
PACIFIC JOURNAL OF MATHEMATICS
}

Founded in 1951 by E. F. Beckenbach (1906-1982) and F. Wolf (1904-1989)

$$
\text { msp.org/pjm }
$$

\section{EDITORS}

\author{
Don Blasius (Managing Editor) \\ Department of Mathematics \\ University of California \\ Los Angeles, CA 90095-1555 \\ blasius@math.ucla.edu
}

\author{
Paul Balmer \\ Department of Mathematics \\ University of California \\ Los Angeles, CA 90095-1555 \\ balmer@math.ucla.edu \\ Robert Finn \\ Department of Mathematics \\ Stanford University \\ Stanford, CA 94305-2125 \\ finn@math.stanford.edu \\ Sorin Popa \\ Department of Mathematics \\ University of California \\ Los Angeles, CA 90095-1555 \\ popa@math.ucla.edu
}

\author{
Vyjayanthi Chari \\ Department of Mathematics \\ University of California \\ Riverside, CA 92521-0135 \\ chari@math.ucr.edu \\ Kefeng Liu \\ Department of Mathematics \\ University of California \\ Los Angeles, CA 90095-1555 \\ liu@math.ucla.edu \\ Igor Pak \\ Department of Mathematics \\ University of California \\ Los Angeles, CA 90095-1555 \\ pak.pjm@gmail.com \\ Paul Yang \\ Department of Mathematics \\ Princeton University \\ Princeton NJ 08544-1000 \\ yang@math.princeton.edu
}

\section{PRODUCTION}

Silvio Levy, Scientific Editor, production@msp.org

\section{SUPPORTING INSTITUTIONS}

ACADEMIA SINICA, TAIPEI

CALIFORNIA INST. OF TECHNOLOGY

STANFORD UNIVERSITY

UNIV. OF BRITISH COLUMBIA

UNIV. OF CALIFORNIA, BERKELEY

UNIV. OF CALIFORNIA, DAVIS

UNIV. OF CALIFORNIA, LOS ANGELES

UNIV. OF CALIFORNIA, RIVERSIDE

UNIV. OF CALIFORNIA, SAN DIEGO

UNIV. OF CALIF., SANTA BARBARA
KEIO UNIVERSITY

MATH. SCIENCES RESEARCH INSTITUTE

NEW MEXICO STATE UNIV.

OREGON STATE UNIV.
Daryl Cooper

Department of Mathematics

University of California

Santa Barbara, CA 93106-3080 cooper@math.ucsb.edu

Jiang-Hua Lu

Department of Mathematics

The University of Hong Kong

Pokfulam Rd., Hong Kong

jhlu@maths.hku.hk

$$
\text { Jie Qing }
$$

Department of Mathematics

University of California

Santa Cruz, CA 95064

qing@ cats.ucsc.edu

\author{
UNIV. OF CALIF., SANTA CRUZ \\ UNIV. OF MONTANA \\ UNIV. OF OREGON \\ UNIV. OF SOUTHERN CALIFORNIA \\ UNIV. OF UTAH \\ UNIV. OF WASHINGTON \\ WASHINGTON STATE UNIVERSITY
}

These supporting institutions contribute to the cost of publication of this Journal, but they are not owners or publishers and have no responsibility for its contents or policies.

See inside back cover or msp.org/pjm for submission instructions.

The subscription price for 2016 is US $\$ 440 /$ year for the electronic version, and \$600/year for print and electronic.

Subscriptions, requests for back issues and changes of subscriber address should be sent to Pacific Journal of Mathematics, P.O. Box 4163, Berkeley, CA 94704-0163, U.S.A. The Pacific Journal of Mathematics is indexed by Mathematical Reviews, Zentralblatt MATH, PASCAL CNRS Index, Referativnyi Zhurnal, Current Mathematical Publications and Web of Knowledge (Science Citation Index).

The Pacific Journal of Mathematics (ISSN 0030-8730) at the University of California, c/o Department of Mathematics, 798 Evans Hall \#3840, Berkeley, CA 94720-3840, is published twelve times a year. Periodical rate postage paid at Berkeley, CA 94704, and additional mailing offices. POSTMASTER: send address changes to Pacific Journal of Mathematics, P.O. Box 4163, Berkeley, CA 94704-0163.

PJM peer review and production are managed by EditFLOW ${ }^{\circledR}$ from Mathematical Sciences Publishers.

PUBLISHED BY

\section{I. mathematical sciences publishers}

nonprofit scientific publishing

http://msp.org/

(C) 2016 Mathematical Sciences Publishers 


\section{PACIFIC JOURNAL OF MATHEMATICS}

Volume $284 \quad$ No. $1 \quad$ September 2016

Bitwist manifolds and two-bridge knots

JAmes W. CANNON, William J. Floyd, LEeR LAMbert,

WALTER R. PARry and Jessica S. PurCELL

Recognizing right-angled Coxeter groups using involutions

Charles Cunningham, Andy Eisenberg, Adam Piggott and KIM RUANE

On Yamabe-type problems on Riemannian manifolds with boundary

Marco Ghimenti, Anna Maria Micheletti and Angela

PISTOIA

Quantifying separability in virtually special groups

MARK F. HAGEN and PRIYAM PATEL

Conformal designs and minimal conformal weight spaces of vertex operator superalgebras

TOMONORI HASHIKAWA

Coaction functors

S. KALiszewski, Magnus B. LANDSTAD and John QuigG

Cohomology and extensions of braces

VICTORIA LEBED and LEANDRO VENDRAMIN

Noncommutative differentials on Poisson-Lie groups and pre-Lie algebras

SHAHN MAJID and WEN-QING TAO 